\title{
Implications of COVID-19 in Neurological Disorders
}

\author{
Satish Gaddam ${ }^{\mathrm{a}, \mathrm{b}, \mathrm{c}}$
}

\begin{abstract}
The coronavirus disease 2019 (COVID-19) pandemic affected healthcare at different levels and almost all medical specialties are impacted one way or the other. In this review we try to discuss the impact of COVID-19 on neurology in particular and suggest changes that can be adopted in different neurologic diseases for navigating this pandemic without compromising the healthcare delivery to this specific patient population.
\end{abstract}

Keywords: COVID-19 neurology; Neurological disorders; Nervous system; Stroke

\section{Introduction}

Like most medical disciplines, neurology as well, is impacted by coronavirus disease 2019 (COVID-19) pandemic. The direct attack of the nervous system by the coronavirus does not seem to be in the foreground, but a neuro-invasive potential exists. In addition, many patients with neurological conditions are considered to be "at risk" patients with coronavirus infection; monitoring and treatment of these patients must, therefore, adapt to the current situation.

More and more scientific data suggest that the severe acute respiratory syndrome coronavirus 2 (SARS-CoV-2) which currently prevails all over the world would also infect the brain. Certain symptoms observed by doctors could indeed stem from brain dysfunction, but investigations are still underway to better understand this potential neurological damage of COVID-19.

\section{The Neurotropism of Coronaviruses}

Although coronaviruses are essentially respiratory viruses, several studies have reported their ability to also infect the

Manuscript submitted July 19, 2020, accepted August 1, 2020

Published online August 25, 2020

aBaylor Scott \& White Medical Center-Centennial Hospital, Frisco, TX 75035, USA

${ }^{\mathrm{b}}$ Lonestar Neurology, Frisco, TX 75035, USA

${ }^{\mathrm{c} C}$ Corresponding Author: Satish Gaddam, MD (Neurology \& Movement Disorders), Baylor Scott \& White Medical Center-Centennial Hospital, Frisco, TX 75035, USA. Email: drsatishgaddam@gmail.com

doi: https://doi.org/10.14740/jnr617 central nervous system and cause neurological disorders [1].

The neurotropism of coronaviruses is known, in particular for the beta-coronavirus family to which SARS-CoV, Middle East respiratory syndrome coronavirus (MERS-CoV) and SARS-CoV-2 belong which is currently spreading. The neurons of the central nervous system are often the cells targeted by the virus which causes their degeneration. There is $91 \%$ homology between this strain and a human beta-coronavirus, human coronavirus OC43 ( $\mathrm{HCoV}-\mathrm{OC} 43)$, responsible for colds [2].

\section{Is the Nervous System Affected by the Corona- virus?}

The SARS-CoV-2, responsible for COVID-19, belongs to the family of coronaviridae. Cases of neurological damage by other coronaviruses (acute flaccid paralysis [3], encephalitis [4]) are known and neurological damage is expected with SARS$\mathrm{CoV}-2$ as well as its genetic sequence being almost $80 \%$ similar to that of SARS-CoV-1 (epidemics of 2002 and 2003) [5]. In addition to infected cells, SARS-CoV-2 binds to membrane receptors angiotensin-converting enzyme 2 (ACE2), present in the pulmonary and vascular endothelium and also at neuronal level. One of the preferred pathways for entry of the virus into the brain would be retrograde neuronal dissemination via the olfactory mucosa, and then the olfactory bulb [6]. Invasion of the central nervous system has been demonstrated in rodents after inoculation of $\mathrm{CoV}-\mathrm{OC} 43$ (another coronavirus) in the nasal mucosa; the hematogenous route direct or via myeloid cells is also possible [7]. The same coronavirus (CoV-OC43) has been found in the brains of patients with multiple sclerosis (MS); however, these data remain controversial and their role undetermined [8].

The path taken by coronaviruses is not precisely known, but it seems that viruses use synaptic pathways to pass from the cardiorespiratory center to the spinal cord. The mechanoreceptors and chemoreceptors of peripheral nerve endings that are found in the lower respiratory tract are believed to be the gateway through which the virus reaches the central nervous system.

The neurological symptoms of COVID-19 affect only a minority of people: $8 \%$ suffer from headaches and $1 \%$ from nausea and vomiting. In contrast, a study of 240 patients with COVID-19 described neurological manifestations such as loss of consciousness and acute cerebrovascular disorders in $88 \%$ of the severe cases studied [9].

The SARS-CoV-2 virus, responsible for COVID-19 dis- 
ease, uses a receptor to cling and enter the cells of its host in order to multiply. Researchers have shown that this receptor, called ACE2, is expressed by cells of the lungs, heart, kidneys and intestines, and also by neurons and glial cells of the brain. This is why doctors are questioning a potential brain infection with the virus, which can lead to neurological damage in patients [10].

Currently, cases of direct neurological damage by SARS$\mathrm{CoV}-2$ have been reported. The respiratory worsening observed in patients sometimes without comorbidities could be explained by an attack of the cardiorespiratory centers, in particular the ambiguous and solitary nuclei [11]. Anosmia (attack of the olfactory bulb, which is part of the nervous system) is a reported symptom in SARS-CoV-2 patients, as in SARS-CoV [6]. Post-viral Guillain-Barre type neuroses or acute disseminated encephalomyelitis (ADEM) have been reported following infection with other coronaviruses; to date, for COVID-19, cases of parainfectious myelitis, Guillain-Barre syndrome and acute necrotizing hemorrhagic encephalitis have also been reported [7-9]. In addition, in a series of patients suffering from severe forms of COVID-19 with acute respiratory syndrome, 58 of them suffered from neurological damage, mainly encephalopathy, confusion and corticospinal signs [12].

In COVID cases with neurological damage, an analysis of the cerebrospinal fluid including a polymerase chain reaction (PCR) for SARS-CoV-2 seems essential during this period in order to distinguish a direct viral infection from a secondary autoimmune reaction.

\section{Management of Neurological Diseases During the COVID-19 Pandemic}

\section{MS}

MS is not a disease that weakens the immune system; patients with MS are not considered to be "at risk" (infection and/or more serious) for COVID-19 unless they benefit from a basic treatment which modulates the immune response and/or in the presence of other risk factors.

Treatment for a flare-up should be evaluated on a caseby-case basis, as high doses of cortisone temporarily increase the risk of infection. Continued on-going disease modifying therapy is recommended, as stopping it could lead to worsening of MS. First-line injectable treatments (glatiramer acetate or interferon-beta) and natalizumab would not be associated with a risk for COVID-19 [13]. Oral treatments could reduce the immune responses to COVID-19: fingolimod, known for a risk of respiratory tract infections and dimethyl fumarate or teriflunomide, especially if the number of lymphocytes is reduced. "Pulsed" therapies (cladribine or alemtuzumab) and anti-CD20 (rituximab or ocrelizumab) would increase the risk of severe infection. For these treatments, we propose to assess the situation for each patient. For anti-CD20, the decision to repeat an infusion could be based on the residual number of memory B cells $\left(\mathrm{CD} 19^{+}, \mathrm{CD} 27^{+}\right)$as proposed for neuromyelitis optica (NMO) [14]. During the COVID-19 epidemic, in case of inactive MS, the introduction of a new basic therapy may be delayed or a first-line injectable treatment proposed. In the case of severe MS, natalizumab is to be preferred. Stem cell transplant treatment is contraindicated.

However, the risk of a more severe COVID-19 infection undergoing MS background therapy remains theoretical, as the severe complications of COVID-19 may be secondary to an exaggerated immune response, in particular cytokine release syndrome.

In fact, it has been hypothesized that immunomodulatory treatments used in MS may be beneficial in this "hyperimmune" phase of COVID-19. Thus, clinical trials are underway to treat these complications of COVID-19 with basic MS treatments, such as interferons-beta (NCT04315948) or fingolimod (NCT04280588).

\section{Stroke}

At the start of the epidemic, there is a decrease in hospitalizations for acute stroke and other urgent pathologies $[15,16]$, probably linked to the reduction in ambulatory consultations and the reassignment of specialized units (emergency, stroke unit, intensive care, and radiology) to treat COVID-19 patients.

A large proportion of stroke patients belong to the population at risk for COVID-19, so it is essential to maintain access to dedicated care for these patients. In addition, there are several potential links between coronavirus and stroke: coagulopathy, heart damage and neurotropic capacities of the virus [17-19]. In two Chinese retrospective series, approximately $6 \%$ of COVID-19 patients developed stroke, cerebral venous thrombosis or cerebral hemorrhage, within 10 days of the first symptoms. These patients were older, had more cardiovascular comorbidities, and more severe pneumonia $[9,20]$.

\section{Epilepsy}

Epilepsy does not seem to put patients at particular risk [21]. Similarly, no antiepileptic treatment has any effect on the immune system, except in rare situations (adrenocorticotropic hormone (ACTH) or steroids for West syndrome and everolimus in Bourneville's sclerosis tuberose). It is therefore important to remind patients to take their treatment regularly and to be careful not to run out of medication. The monitoring of patients deemed at risk by the public health officials should possibly be done via telemedicine.

The COVID-19 epidemic does not appear to be accompanied by acute symptomatic attacks [22]. In contrast, the fever caused by COVID-19 could increase the risk of an attack in patients with epilepsy. Protocols for treating crises in patients sensitive to fever could be discussed.

\section{Neurodegenerative diseases}

The majority of people with neurodegenerative diseases are elderly and must de facto be considered "vulnerable". Adher- 
ence to the hygiene rules recommended by the public health officials is crucial in this category of the population [23]. It is important that patients remain active at home despite limited access to therapies. In the event of infection, COVID-19 or other, the symptoms of the disease are conventionally worsened, sometimes complicated by a confused state and falls. The autonomy can be degraded; self-isolation seems hardly possible in these patients putting the caregiver(s) helping at risk of contamination.

The medication does not increase the risk of infection and should not be changed without reason. As far as possible, the neurological follow-up of these patients must be ensured by telephone consultation.

\section{Anosmia and ageusia}

Anosmia (loss of smell) and/or ageusia (loss of taste) have been very commonly seen in patients with COVID-19. According to scientists, these symptoms could be minor neurological manifestations. Anosmia, for example, could arise from damage to cells surrounding olfactory neurons. If so, the olfactory bulb could be a gateway for the virus to the brain. Other investigations must be carried out to verify this hypothesis and to know if these symptoms are always reversible or if there are cases of permanent damage [24].

\section{A brainstem attack}

Scientists have speculated that the breathing difficulties seen in patients with COVID-19 could be caused by brain damage in addition to the lung. This region, located between the brain and the spinal cord, contains the breathing control centers. This hypothesis is based on work in animals showing that previously studied coronaviruses, such as SARS-CoV, can spread to the brainstem from the lower respiratory tract [11].

\section{Encephalopathy cases}

In some patients, the disease can manifest itself in a neurological form. A few cases of encephalopathy have been identified in the USA in patients who tested positive for SARS-CoV-2. This rare complication of viral infection is not directly due to the presence of the virus in the brain but to a massive release of inflammatory molecules, cytokines, produced by the immune system to fight the virus and which would weaken the blood-brain barrier, essential to isolate the brain from toxic substances [25].

\section{Conclusions}

COVID-19 pandemic is impacting every aspect of healthcare and neurology is no exception. In this article we tried to review available literature and the implications of this novel virus on different neurologic diseases and also its direct impact on the nervous system. As new data keep emerging, it is paramount to be abreast of these new changes and to adapt to those changes as we sail through this pandemic to ensure the best possible care for patients.

\section{Acknowledgments}

None to declare.

\section{Financial Disclosure}

None to declare.

\section{Conflict of Interest}

None to declare.

\section{Data Availability}

The author declares that data supporting the findings of this study are available within the article.

\section{References}

1. Baig AM. Neurological manifestations in COVID-19 caused by SARS-CoV-2. CNS Neurosci Ther. 2020;26(5):499-501.

2. Filatov A, Sharma P, Hindi F, Espinosa PS. Neurological Complications of Coronavirus Disease (COVID-19): encephalopathy. Cureus. 2020;12(3):e7352.

3. Turgay C, Emine T, Ozlem K, Muhammet SP, Haydar AT. A rare cause of acute flaccid paralysis: Human coronaviruses. J Pediatr Neurosci. 2015;10(3):280-281.

4. Morfopoulou S, Brown JR, Davies EG, Anderson G, Virasami A, Qasim W, Chong WK, et al. Human coronavirus OC43 associated with fatal encephalitis. N Engl J Med. 2016;375(5):497-498.

5. Zhou P, Yang XL, Wang XG, Hu B, Zhang L, Zhang W, Si HR, et al. A pneumonia outbreak associated with a new coronavirus of probable bat origin. Nature. 2020;579(7798):270-273.

6. Huang C, Wang Y, Li X, Ren L, Zhao J, Hu Y, Zhang $\mathrm{L}$, et al. Clinical features of patients infected with 2019 novel coronavirus in Wuhan, China. Lancet. 2020;395(10223):497-506.

7. Desforges M, Le Coupanec A, Dubeau P, Bourgouin A, Lajoie L, Dube M, Talbot PJ. Human coronaviruses and other respiratory viruses: underestimated opportunistic pathogens of the central nervous system? Viruses. 2019;12(1):14.

8. Dessau RB, Lisby G, Frederiksen JL. Coronaviruses in brain tissue from patients with multiple sclerosis. Acta Neuropathol. 2001;101(6):601-604. 
9. Mao L, Jin H, Wang M, Hu Y, Chen S, He Q, Chang J, et al. Neurologic manifestations of hospitalized patients with coronavirus disease 2019 in Wuhan, China. JAMA Neurol. 2020.

10. Baig AM, Khaleeq A, Ali U, Syeda H. Evidence of the COVID-19 virus targeting the CNS: tissue distribution, host-virus interaction, and proposed neurotropic mechanisms. ACS Chem Neurosci. 2020;11(7):995-998.

11. Li YC, Bai WZ, Hashikawa T. The neuroinvasive potential of SARS-CoV2 may play a role in the respiratory failure of COVID-19 patients. J Med Virol. 2020;92(6):552555.

12. Suzuki M, Saito K, Min WP, Vladau C, Toida K, Itoh $\mathrm{H}$, Murakami S. Identification of viruses in patients with postviral olfactory dysfunction. Laryngoscope. 2007;117(2):272-277.

13. Toscano G, Palmerini F, Ravaglia S, Ruiz L, Invernizzi P, Cuzzoni MG, Franciotta D, et al. Guillain-Barre syndrome associated with SARS-CoV-2. N Engl J Med. 2020;382(26):2574-2576.

14. Zhao K, Huang J, Dai D, Feng Y, Liu L, Nie S. Acute myelitis after SARS-CoV-2 infection: a case report. medRxiv. 2020;2020.03.16.20035105. http://medrxiv.org/ content/early/2020/03/18/2020.03.16.20035105.abstract.

15. Helms J, Kremer S, Merdji H, Clere-Jehl R, Schenck $\mathrm{M}$, Kummerlen $\mathrm{C}$, Collange $\mathrm{O}$, et al. Neurologic features in severe SARS-CoV-2 Infection. N Engl J Med. 2020;382(23):2268-2270.

16. Cohen JA, Tenenbaum N, Bhatt A, Zhang Y, Kappos L. Extended treatment with fingolimod for relapsing multiple sclerosis: the 14-year LONGTERMS study results. Ther Adv Neurol Disord. 2019;12:1756286419878324.

17. Lee SY, Khang YH, Lim HK. Impact of the 2015 Middle East Respiratory Syndrome outbreak on emergency care utilization and mortality in South Korea. Yonsei Med J. 2019;60(8):796-803.

18. Zhao J, Rudd A, Liu R. Challenges and Potential Solutions of Stroke Care During the Coronavirus Disease 2019 (COVID-19) Outbreak. Stroke. 2020;51(5):13561357.

19. Blackburn R, Zhao H, Pebody R, Hayward A, WarrenGash C. Laboratory-confirmed respiratory infections as predictors of hospital admission for myocardial infarction and stroke: time-series analysis of English data for 20042015. Clin Infect Dis. 2018;67(1):8-17.

20. Kim JE, Heo JH, Kim HO, Song SH, Park SS, Park TH, Ahn JY, et al. Neurological complications during treatment of Middle East Respiratory Syndrome. J Clin Neurol. 2017;13(3):227-233.

21. Ruegg S. https://www.epi.ch/fr/mise-a-jour-sur-le-coronavirus-et-lepilepsie/. 2019

22. Lu L, Xiong W, Liu D, Liu J, Yang D, Li N, Mu J, et al. New onset acute symptomatic seizure and risk factors in coronavirus disease 2019: A retrospective multicenter study. Epilepsia. 2020;61(6):e49-e53.

23. Guan WJ, Ni ZY, Hu Y, Liang WH, Ou CQ, He JX, Liu $\mathrm{L}$, et al. Clinical Characteristics of Coronavirus Disease 2019 in China. N Engl J Med. 2020;382(18):1708-1720.

24. Lechien JR, Chiesa-Estomba CM, De Siati DR, Horoi M, Le Bon SD, Rodriguez A, Dequanter D, et al. Olfactory and gustatory dysfunctions as a clinical presentation of mild-to-moderate forms of the coronavirus disease (COVID-19): a multicenter European study. Eur Arch Otorhinolaryngol. 2020;277(8):2251-2261.

25. Poyiadji N, Shahin G, Noujaim D, Stone M, Patel S, Griffith B. COVID-19-associated acute hemorrhagic necrotizing encephalopathy: imaging features. Radiology. 2020;296(2):E119-E120. 